\title{
Relación del índice nutricional pronóstico con complicaciones y mortalidad en los pacientes con cáncer gástrico sometidos a gastrectomía en un hospital de tercer nivel de Bogotá, Colombia
}

\author{
Relationship of the prognostic nutritional index with complications and \\ mortality in patients with gastric cancer who underwent gastrectomy in \\ a tertiary hospital in Bogotá, Colombia
}

Andrés Eduardo Marín-Castro ${ }^{1} \mathbb{D}$, Douglas Omar Ortiz-Espinel ${ }^{2}$ (D), Carlos Alberto Sánchez-Toro ${ }^{\mathbb{D}}$, Carlos Manuel Zapata-Acevedo ${ }^{4}$ D , María José Marín-Castro ${ }^{5} \mathbb{D}$,

Blas Darío Conde-Rodríguez ${ }^{6} \mathbb{D}$, Gerardo Ardila-Duarte ${ }^{7} \mathbb{D}$

1 Médico, Residente de Cirugía general, Universidad Militar Nueva Granada, Hospital Universitario Clínica San Rafael, Bogotá, D.C., Colombia.

2 Médico, Especialista en Cirugía general, Gastroenterología y Laparoscopia avanzada, Hospital Universitario Clínica San Rafael; Coordinador académico, programa de Cirugía general, Universidad Militar Nueva Granada, Bogotá, D.C., Colombia.

3 Médico, especialista en Cirugía general; jefe de Servicio de Cirugía, Hospital Universitario Clínica San Rafael; Coordinador programa de Cirugía general, Universidad Militar Nueva Granada, Bogotá, D.C., Colombia.

4 Médico, especialista en Cirugía general, Hospital Universitario de La Samaritana; Coordinador programa de Cirugía general, Universidad Nacional de Colombia, Hospital Universitario Nacional, Bogotá, D.C., Colombia.

5 Estudiante de Medicina, Universidad Pontificia Bolivariana, Medellín, Colombia.

6 Estudiante de Medicina, Universidad El Bosque, Bogotá, D.C., Colombia.

7 Matemático, Magister en Estadística; profesor asociado, Universidad Militar Nueva Granada, Bogotá, D.C., Colombia.

\section{Resumen}

Introducción. El cáncer gástrico es el quinto cáncer diagnosticado con mayor frecuencia y la tercera causa de muerte por cáncer en el mundo. En el tratamiento quirúrgico, la evidencia actual apoya las medidas preoperatorias e índices pronósticos para mejorar la supervivencia. El índice nutricional pronóstico, que une los valores de los linfocitos circulantes en sangre periférica con los de la albúmina sérica, ha presentado características de ser un marcador nutricional e inmunológico con valor predictivo sobre complicaciones y mortalidad. El objetivo de este estudio fue determinar la relación entre el índice nutricional pronóstico con las complicaciones y mortalidad en pacientes con cáncer gástrico sometidos a gastrectomía.

Métodos. Se llevó a cabo un estudio observacional descriptivo, de corte transversal, con componente analítico, mediante la revisión retrospectiva de las historias clínicas.

Fecha de recibido: 11/06/2021 - Fecha de aceptación: 07/09/2021 - Publicación en línea: 07/10/2021

Correspondencia: Andrés Eduardo Marín-Castro, Carrera 8 \# 17-45 Sur, Bogotá, D.C., Colombia. Teléfono: 3146155679

Correo electrónico: andrese9@hotmail.com

Citar como: Marín-Castro AE, Ortiz-Espinel DO, Sánchez-Toro CA, Zapata-Acevedo CM, Marín-Castro MJ, Conde-Rodríguez BD, Ardila-Duarte G. Relación del índice nutricional pronóstico con complicaciones y mortalidad en los pacientes con cáncer gástrico sometidos a gastrectomía en un hospital de tercer nivel de Bogotá, Colombia. Rev Colomb Cir. 2022;37:60-71. https://doi.org/10.30944/20117582.951

Este es un artículo de acceso abierto bajo una Licencia Creative Commons - BY-NC-ND https://creativecommons.org/licenses/by-nc-nd/4.0/deed.es 
Resultados. Se analizaron 113 pacientes sometidos a gastrectomía total o subtotal. Se encontró asociación entre el índice nutricional pronóstico y la mortalidad; todos los pacientes que murieron tenían un índice menor o igual a 46. También se encontró asociación inversa entre el valor del índice y la presentación de complicaciones posoperatorias, como sepsis, peritonitis, fuga de la anastomosis y sangrado.

Discusión. Similar a nuestro análisis, varios estudios plantean que un índice nutricional pronóstico bajo podría tener un valor predictivo sobre la frecuencia de complicaciones y supervivencia global en pacientes con cáncer gástrico llevados a cirugía.

Conclusión. El índice nutricional pronóstico se asocia con la mortalidad y complicaciones posoperatorias en pacientes sometidos a gastrectomía por cáncer gástrico.

Palabras clave: neoplasias gástricas; evaluación nutricional; gastrectomía; complicaciones posoperatorias; mortalidad; pronóstico.

\begin{abstract}
Introduction. Gastric cancer is the fifth most frequently diagnosed cancer and the third cause of cancer death in the world. In surgical treatment, current evidence supports preoperative measures and prognostic index to improve survival. The prognostic nutritional index, which unites the values of circulating lymphocytes in peripheral blood with those of serum albumin, has presented characteristics of being a nutritional and immunological marker with predictive value on complications and mortality. The objective of this study was to determine the relationship between the prognostic nutritional index with complications and mortality in patients with gastric cancer undergoing gastrectomy.
\end{abstract}

Methods. A descriptive, cross-sectional, observational study with an analytical component was carried out by a retrospective review of medical records.

Results. A total of 113 patients who underwent total or subtotal gastrectomy were analyzed. An association was found between mortality and the prognostic nutritional index and mortality; all patients who died had an index $\leq 46$. An inverse association was also found between the value of the index and the presentation of postoperative complications, such as sepsis, peritonitis, anastomotic leak, and bleeding.

Discussion. Similar to our analysis, several studies suggest that a low prognostic nutritional index could have a predictive value on the frequency of complications and overall survival in patients with gastric cancer undergoing surgery.

Conclusion. The prognostic nutritional index is associated with mortality and postoperative complications in patients undergoing gastrectomy for gastric cancer.

Keywords: stomach neoplasms; nutrition assessment; gastrectomy; postoperative complications; mortality; prognosis.

\section{Introducción}

En los últimos años, la incidencia de cáncer gástrico (CG) ha disminuido, no obstante, y a pesar de las mejoras en los tratamientos quirúrgicos y oncológicos, la mortalidad sigue siendo muy elevada ${ }^{1-5}$. Existen diferencias en la incidencia del CG de acuerdo con su distribución geográfica: las zonas de más alta incidencia son Asia (principalmente Japón), oeste de Sur América y Europa del este. También hay una notable variabilidad en la historia natural de la enfermedad entre los pacientes, sobre todo en la supervivencia. La tasa de supervivencia a los 5 años del diagnóstico es del 20-30\% en los países occidentales ${ }^{6-12}$. Colombia 
está entre los países con tasa de incidencia más alta (17,4 a 48,2 por 100.000 habitantes), y aquí el CG es la primera causa de muerte por cáncer en hombres y la tercera en las mujeres, por lo que este tumor representa un verdadero problema de salud pública.

El tratamiento del CG se basa fundamentalmente en una cirugía radical ${ }^{13}$. Para conseguirla hay que valorar en primer lugar si el paciente está en condiciones de ser operado y si el tumor es resecable. La resecabilidad, en el momento del diagnóstico, tan solo puede presumirse mediante la clasificación TNM clínica, cuya precisión es limitada y tiende a la infra-estadificación en al menos un $20 \%$ de los casos, por lo que es un criterio que sólo se confirma tras la cirugía. Por eso, se precisa disponer de otros parámetros predictivos, independientes del TNM clínico, y que se puedan determinar en el momento del diagnóstico del tumor, como la inmunidad, inflamación y nutrición ${ }^{12,14}$.

El paciente con CG presenta muchos síntomas que en última instancia lo llevan a un estado de desnutrición ${ }^{14}$; el rápido crecimiento de las células tumorales malignas provoca el consumo de gran cantidad de nutrientes, falta de síntesis de nutrientes, necrosis y producción de sustancias tóxicas, que conducen a trastornos metabólicos, por lo tanto, la mayoría de los pacientes tienen diferentes grados de desnutrición en el momento del tratamiento ${ }^{15,16}$. La desnutrición puede retrasar el tratamiento, lo que puede llevar a que la enfermedad progrese. Además, la desnutrición conduce a la disminución de la función de las células T y al deterioro del ambiente intestinal, agravando aún más la progresión de la inflamación asociada a tumor ${ }^{17}$. En 1863, Virchow propuso por primera vez la asociación entre inflamación y cáncer, y desde entonces, el papel de la inflamación en la génesis del tumor, desarrollo tumoral y las metástasis ha sido explorada continuamente ${ }^{18}$. Mantovani et al ${ }^{19}$ mostraron que la inflamación asociada al tumor se caracteriza por la liberación de factores inflamatorios, que favorecen aún más el desarrollo del tumor, la invasión y las metástasis ${ }^{20-23}$.

La desnutrición causa cambios en el peso corporal, la masa muscular, los niveles de albúmina sérica, conteo de linfocitos, el número de células
T ayudadoras, niveles de interleucinas y respuesta blastogénica. Las consecuencias acumulativas de estas alteraciones afectan adversamente el pronóstico de los pacientes con cáncer, a través del aumento de las complicaciones postoperatorias, atribuido a la vulnerabilidad del tejido, la cicatrización de la herida deteriorada, la susceptibilidad a la infección y la progresión tumoral acelerada ${ }^{24}$.

El Índice Nutricional Pronóstico (INP) fue establecido por primera vez por académicos japoneses y fue utilizado originalmente para evaluar el estado de nutrición preoperatorio, riesgo quirúrgico y complicaciones postoperatorias en pacientes quirúrgicos ${ }^{1}$. El Índice de Onodera ${ }^{10}$, también denominado INP, combina los valores de los linfocitos circulantes y los de la albúmina sérica. Se ha propuesto como predictor de pronóstico para pacientes con neoplasias gastrointestinales ${ }^{25}$, cáncer colorrectal ${ }^{26}$, cáncer de hígado ${ }^{27}$ y cáncer de páncreas ${ }^{28}$; reflejando la inmunocompetencia y estado nutricional con mayor precisión ${ }^{24}$.

El indice pronóstico nutricional de Onodera (Onodera's prognostic nutricional index, OPNI) se calcula con la siguiente formula ${ }^{29}$ :

OPNI = $10 \mathrm{X}$ Albumina (g/Dl) + 0,005 X linfocitos circulantes / mm 3

Ouyang et al ${ }^{5}$ mostraron que los niveles de albúmina sérica preoperatorio están asociados con el pronóstico en pacientes con CG. La respuesta inmune de los linfocitos al tumor se ha aplicado gradualmente a la evaluación pronóstica en los pacientes con cáncer en los últimos años ${ }^{25}$. Un estudio reciente muestra que el INP preoperatorio es superior a los marcadores inflamatorios tradicionales para la evaluación del pronóstico después de la gastrectomía y que es un factor de riesgo independiente para mortalidad después de la gastrectomía ${ }^{30}$. Esto puede ser explicado porque el INP preoperatorio refleja el estado nutricional y la respuesta inflamatoria del cuerpo.

El objetivo de este estudio fue determinar la relación entre el INP preoperatorio con las complicaciones, reintervenciones y mortalidad en los pacientes con cáncer gástrico sometidos a gastrectomía. 


\section{Métodos}

Se realizó un estudio observacional descriptivo, de corte transversal, con componente analítico, donde se incluyeron los pacientes con cáncer gástrico sometidos a gastrectomía total o subtotal, entre los años 2014 a 2019, en el Hospital Universitario Clínica San Rafael de la ciudad de Bogotá, D.C., Colombia. Se excluyeron los pacientes que no contaran con una historia clínica completa, sin posibilidad de completar los datos, que no aceptaron ser entrevistados telefónicamente cuando la recolección de los datos lo ameritaba, aquellos que tuvieran una neoplasia sincrónica o metacrónica, con alguna enfermedad infecciosa o autoinmune, o con consumo crónico de esteroides.

Se realizó un muestreo probabilístico con una confianza del $95 \%$, una potencia del $90 \%$, un error del $5 \%$. El tamaño de la muestra se calculó en 113 pacientes, que fueron seleccionados de acuerdo con la secuencia aleatoria. La fuente de información y unidad de análisis fue la historia clínica. Para la recolección y consignación de las variables del estudio, se diseñó un formulario de recolección de datos, una base de datos y un libreto telefónico.

Se realizó un análisis exploratorio de los datos para describir la muestra, donde las variables discretas se mostraron en porcentajes y tamaño de muestra y las variables continuas se presentaron en promedio, mediana, error estándar y tamaño de muestra. Se aplicó la prueba de Shapiro-Wilk, para determinar si las variables continuas presentaban distribución normal; prueba de Chi cuadrado o test exacto de Fisher, para determinar la relación de las variables discretas y el INP, con sus OR e intervalos de confianza; se calculó correlación de Spearman-Pearson, para determinar la relación de las variables continuas con el INP, regresión logística múltiple, con la cual se encontraron las variables relacionadas con las complicaciones y curva de Kapplan-Meyer entre los pacientes que presentaron mortalidad con la prueba de Wilcoxon.

\section{Resultados}

Se incluyeron 113 pacientes sometidos a gastrectomía total o subtotal por cáncer gástrico, cuyas características clínicas y patológicas se presentan en la tabla 1. La edad media de la cohorte de estudio fue de 64 años y la mayoría de los pacientes eran del género masculino. El 38,9\% de los pacientes tenían comorbilidades cardiovasculares. $\mathrm{Al} 63,7 \%$ de los pacientes se les realizó gastrectomía total y la mitad recibieron quimioterapia neoadyuvante. En el 39,8 \% de los pacientes se presentó algún tipo de complicación, con la necesidad de cirugía adicional en el 25 \% de los casos.

Las características nutricionales de los pacientes se muestran en la tabla 2 y los exámenes de laboratorio en la tabla 3. La mayoría de los pacientes $(61,1 \%)$ tenían un grado de nutrición clasificado como peso saludable. Los pacientes perdieron en promedio casi ocho kilogramos de peso en los últimos 6 meses y hasta la mitad de los pacientes perdieron siete kilogramos de peso. La mayoría de los pacientes $(67,3 \%)$ recibieron nutrición parenteral y casi la mitad de los pacientes nutrición enteral. En relación con el INP, hasta la mitad de los pacientes tenían un puntaje de 38,4.

Tabla 1. Características clínico-patológicas de los pacientes sometidos a gastrectomía ( $n=113$ pacientes).

\begin{tabular}{lc}
\hline Variable & Frecuencia (\%) \\
\hline Edad, media (desviación estándar) & $64(1,3)$ \\
Sexo & \\
Masculino & $62(54,9)$ \\
Femenino & $51(45,1)$ \\
Comorbilidades & $44(38,9)$ \\
Diabetes mellitus 2 & $17(15)$ \\
Hipertensión arterial & $37(32,7)$ \\
Dislipidemia & $3(2,7)$ \\
Extensión de la resección & \\
Total & $72(63,7)$ \\
Subtotal & $41(36,3)$ \\
Neoadyuvancia & $58(51,3)$ \\
Complicaciones & $45(39,8)$ \\
Sangrado & $12(10,6)$ \\
Peritonitis & $10(8,8)$ \\
Fuga de la anastomosis & $23(20,4)$ \\
Sepsis & $31(27,4)$ \\
Cirugías adicionales & \\
4 & $3(2,7)$ \\
3 & $2(1,8)$ \\
2 & $11(9,7)$ \\
1 & $29(25,7)$ \\
\hline
\end{tabular}


Tabla 2. Características nutricionales de los pacientes sometidos a gastrectomía

( $n=113$ pacientes).

\begin{tabular}{lc}
\hline Variable & Frecuencia (\%) \\
\hline Peso $(\mathrm{kg})$, media (desviación estándar) & $59,2(1,3)$ \\
Talla $(\mathrm{m})$, media (desviación estándar) & $1,6(0)$ \\
Índice de masa corporal $\left(\mathrm{kg} / \mathrm{m}^{2}\right)$, media & $23(0,5)$ \\
(desviación estándar) & \\
Grado de nutrición & \\
Delgadez muy severa & $2(1,8)$ \\
Delgadez severa & $2(1,8)$ \\
Delgadez & $14(12,4)$ \\
Peso saludable & $69(61,1)$ \\
Sobrepeso & $17(15)$ \\
Obesidad grado 1 & $5(4,4)$ \\
Obesidad grado 2 & $3(2,7)$ \\
Obesidad grado 3 & $0(0)$ \\
Obesidad grado 4 & $1(0,9)$ \\
Pérdida de peso en los últimos 6 meses, & $7,9(0,6)$ \\
media (desviación estándar) & $76(67,3)$ \\
Nutrición parenteral & $55(48,7)$ \\
Nutrición enteral & $37,7(0,7)$ \\
Índice Nutricional Pronóstico (INP), media & \\
(desviación estándar) & \\
\hline
\end{tabular}

Tabla 3. Características paraclínicas de los pacientes sometidos a gastrectomía ( $n=113$ pacientes).

\begin{tabular}{|c|c|c|c|}
\hline Variable & Media & DE & Mediana \\
\hline $\begin{array}{l}\text { Recuento de leucocitos } \\
\left(\mathrm{cel} / \mathrm{mm}^{3}\right)\end{array}$ & 8451,5 & 551,3 & 7400,0 \\
\hline Proporción de neutrófilos (\%) & 70,1 & 1,1 & 71,0 \\
\hline Proporción de linfocitos (\%) & 12,2 & 0,7 & 11,0 \\
\hline $\begin{array}{l}\text { Recuento absoluto de linfocitos } \\
\left(\mathrm{cel} / \mathrm{mm}^{3}\right)\end{array}$ & 949,0 & 64,0 & 835,9 \\
\hline Hemoglobina (gr/l) & 11,5 & 0,3 & 11,8 \\
\hline Plaquetas $\left(\mathrm{cel} / \mathrm{mm}^{3}\right)$ & $317.293,2$ & 9870,3 & $304.000,0$ \\
\hline Nivel de albúmina (gr/dl) & 3,3 & 0,1 & 3,3 \\
\hline Nivel de prealbúmina $(\mathrm{mg} / \mathrm{dl})$ & 15,6 & 0,7 & 14,2 \\
\hline Proteínas totales (gr/dl) & 5,6 & 0,1 & 5,4 \\
\hline Nivel de colesterol total (mg/dl) & 133,0 & 3,7 & 137,0 \\
\hline Triglicéridos (mg/dl) & 103,5 & 3,2 & 103,0 \\
\hline Transferrina (mg/dl) & 245,6 & 6,9 & 253,0 \\
\hline Ferritina (ng/ml) & 34,8 & 2,9 & 31,5 \\
\hline
\end{tabular}

La mitad de los pacientes presentaron tumores ubicados en el antro gástrico o con una clasificación macroscópica Borrmann III, es decir un tumor infiltrante y ulcerado (tabla 4). Los estadios clínicos más frecuentes fueron el IIA, IB y IIB. En relación con la histología del tumor, la mayor proporción eran adenocarcinomas bien
Tabla 4. Características clínicas oncológicas de los pacientes sometidos a gastrectomía $(n=113$ pacientes $)$.

\begin{tabular}{lc}
\hline Variable & Frecuencia (\%) \\
\hline Extensión macroscópica del tumor & \\
Borrmann V & $5(4,4)$ \\
Borrmann IV & $29(25,7)$ \\
Borrmann III & $58(51,3)$ \\
Borrmann II & $21(18,6)$ \\
Etapa clínica del tumor & \\
IA & $10(8,8)$ \\
IB & $18(15,9)$ \\
IIA & $40(35,4)$ \\
IIB & $13(11,5)$ \\
IIIA & $10(8,8)$ \\
IIIB & $4(3,5)$ \\
IIIC & $4(3,5)$ \\
IV & $14(12,4)$ \\
Histología del tumor & \\
Clasificación de la Organización Mundial & \\
de la Salud (WHO) & \\
Adenocarcinoma bien diferenciado & $41(36,3)$ \\
Carcinoma de células en anillo de sello & $32(28,3)$ \\
Adenocarcinoma mal diferenciado & $20(17,7)$ \\
Carcinoma pobremente cohesivo & $5(4,4)$ \\
Adenocarcinoma mucinoso & $5(4,4)$ \\
Otros & $10(8,8)$ \\
Clasificación de Lauren & \\
Intestinal & $66(58,4)$ \\
Difuso & $27(23,9)$ \\
Indeterminado & $20(17,7)$ \\
Localización del tumor & \\
Cardias & $9(8)$ \\
Fondo & $30(26,5)$ \\
Cuerpo & $55(48,7)$ \\
Antro & $11(9,7)$ \\
Píloro & \\
\hline
\end{tabular}

diferenciados e intestinales en la clasificación de Lauren.

Los pacientes tuvieron una hospitalización promedio de 9 días antes de la cirugía y en promedio se identificaron las complicaciones a los 2 días después de la cirugía (tabla 5). El $61 \%$ requirieron Unidad de Cuidados Intensivos, con una estancia promedio de casi cuatro días.

Los factores clínico-patológicos y nutricionales que fueron significativos en el análisis multivariado se incluyeron en la tabla 6. Se encontró asociación significativa entre el INP y la mortalidad; todos los pacientes que murieron tenían un INP menor o igual a 46,03 , y del total de pacientes con un INP menor o igual a 46,03 , murieron el $24,2 \%$. 
Tabla 5. Características relacionadas con la hospitalización.

\begin{tabular}{lc}
\hline Variable & Valor \\
\hline $\begin{array}{l}\text { Días de estancia hospitalaria previa a la } \\
\text { cirugía, media ( } \pm \text { desviación estándar) }\end{array}$ & $9,1( \pm 0,7)$ \\
$\begin{array}{l}\text { Días posquirúrgicos al momento de } \\
\text { identificación complicaciones, media } \\
\text { ( } \pm \text { desviación estándar) }\end{array}$ & $2,1( \pm 0,3)$ \\
$\begin{array}{l}\text { Manejo en Unidad de Cuidados Intensivos } \\
\text { (UCI) }\end{array}$ & $59(61,1 \%)$ \\
$\begin{array}{l}\text { Manejo en Unidad de Cuidados Especiales } \\
\text { (UCE) }\end{array}$ & $22(19,5 \%)$ \\
$\begin{array}{l}\text { Días de estancia en UCl, media } \\
( \pm \text { desviación estándar) }\end{array}$ & $3,9( \pm 0,6)$ \\
$\begin{array}{l}\text { Días de estancia en UCE, media } \\
\text { ( } \pm \text { desviación estándar) }\end{array}$ & $1,1( \pm 0,3)$ \\
$\begin{array}{l}\text { Días de estancia en hospitalización, media } \\
( \pm \text { desviación estándar) }\end{array}$ & $20,6( \pm 1,3)$
\end{tabular}

El análisis y la correlación del INP con las variables continuas se esquematiza en la tabla 7. Se identificó una relación positiva estadísticamente significativa entre el INP con el valor de hemoglobina, prealbúmina, proteínas totales, colesterol total y transferrina, así como una relación inversa entre el INP y los días posquirúrgicos al momento de identificar las complicaciones (figura 1). Para las demás variables no hubo una diferencia estadísticamente significativa, pero se observaron unas tendencias establecidas.

En la figura 2 se muestra la curva de supervivencia, calculada independientemente del valor de INP, en donde se observa que los pacientes tenían una probabilidad del $80 \%$ de sobrevivir hasta dos años.

En la regresión logística se encontró asociación inversa entre el valor de INP y la presentación

Tabla 6. Relación entre el índice nutricional pronóstico (INP) y la mortalidad en los pacientes sometidos a gastrectomía ( $n=113$ pacientes).

\begin{tabular}{|c|c|c|c|c|c|}
\hline \multicolumn{2}{|c|}{ Mortalidad } & \multirow{2}{*}{$\frac{\text { INP } \leq 46,03}{24}$} & \multirow{2}{*}{$\frac{\text { INP }>\mathbf{4 6 , 0 3}}{0}$} & \multirow{2}{*}{$\begin{array}{c}\text { Total } \\
24\end{array}$} & \multirow[t]{2}{*}{$p$} \\
\hline \multirow{4}{*}{ Muerto } & $\mathrm{n}$ & & & & \\
\hline & $\%$ Mortalidad & 100,0 & 0,0 & 100,0 & \\
\hline & INP menor a 46,03 & $24,2 \%$ & $0,0 \%$ & $21,2 \%$ & \\
\hline & $\%$ del total & 21,2 & 0,0 & 21,2 & \multirow{5}{*}{0,038} \\
\hline \multirow{4}{*}{ Vivo } & $\mathrm{n}$ & 75 & 14 & 89 & \\
\hline & $\%$ Mortalidad & 84,3 & 15,7 & 100,0 & \\
\hline & INP menor a 46,03 & $75,8 \%$ & $100,0 \%$ & $78,8 \%$ & \\
\hline & $\%$ del total & 66,4 & $12,4 \%$ & $78,8 \%$ & \\
\hline
\end{tabular}

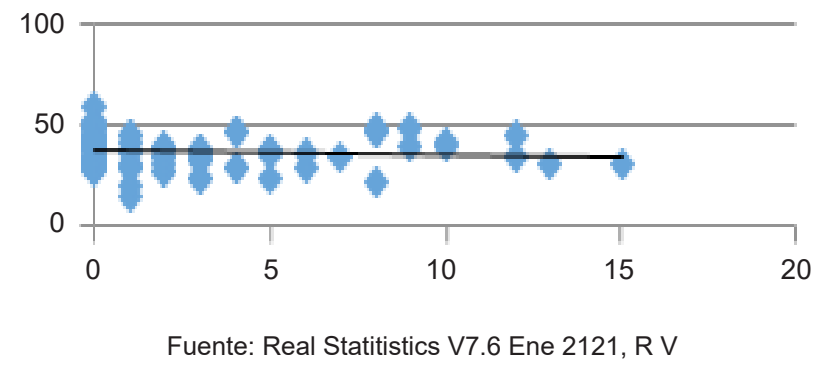

Figura 1. Días posquirúrgicos al momento de identificar las complicaciones. 
Tabla 7. Correlación entre el índice nutricional pronóstico (INP) y las variables continuas en los pacientes sometidos a gastrectomía ( $\mathrm{n}=113$ pacientes).

\begin{tabular}{|c|c|c|c|}
\hline Variable & Sw $p$ * & Rho $(\rho) * *$ & $\mathbf{p}$ \\
\hline Edad (años) & 0,008 & $-0,06$ & 0,518 \\
\hline Peso (kg) & 0,001 & 0,10 & 0,271 \\
\hline Talla (cm) & 0,257 & $-0,04$ & 0,722 \\
\hline Índice de masa corporal & 0,000 & 0,14 & 0,137 \\
\hline Pérdida de peso en los últimos 6 meses & 0,000 & 0,02 & 0,853 \\
\hline Valor de hemoglobina (gr/l) & 0,528 & 0,25 & 0,012 \\
\hline Recuento de leucocitos (cel/mm³) & 0,000 & 0,06 & 0,504 \\
\hline Recuento de neutrófilos (\%) & 0,027 & $-0,12$ & 0,203 \\
\hline Recuento de plaquetas (cel/mm³) & 0,007 & 0,09 & 0,330 \\
\hline Nivel de prealbúmina (mg/dl) & 0,053 & 0,28 & 0,000 \\
\hline Proteínas totales (gr/dl) & 0,015 & 0,48 & 0,000 \\
\hline Colesterol total (mg/dl) & 0,013 & 0,22 & 0,017 \\
\hline Triglicéridos (md/dl) & 0,033 & $-0,07$ & 0,456 \\
\hline Ferritina (ng/ml) & 0,000 & 0,00 & 0,970 \\
\hline Transferrina (mg/dl) & 0,080 & 0,18 & 0,020 \\
\hline Nutrición parenteral (días) & 0,000 & $-0,16$ & 0,093 \\
\hline Nutrición enteral (días) & 0,000 & $-0,09$ & 0,356 \\
\hline Días de estancia hospitalaria previa a la cirugía & 0,000 & $-0,17$ & 0,075 \\
\hline Días posquirúrgicos al momento de identificar complicaciones & 0,000 & $-0,27$ & 0,004 \\
\hline Días de estancia en Unidad de Cuidado Intensivos & 0,000 & $-0,03$ & 0,721 \\
\hline Días de estancia en Unidad Cuidados Especiales & 0,000 & $-0,12$ & 0,210 \\
\hline Días de estancia en hospitalización & 0,000 & 0,00 & 0,974 \\
\hline
\end{tabular}

* Prueba de Shapiro-Wilk, menor de 0,05, distribución no normal

${ }^{* *}$ Correlación de Spearman

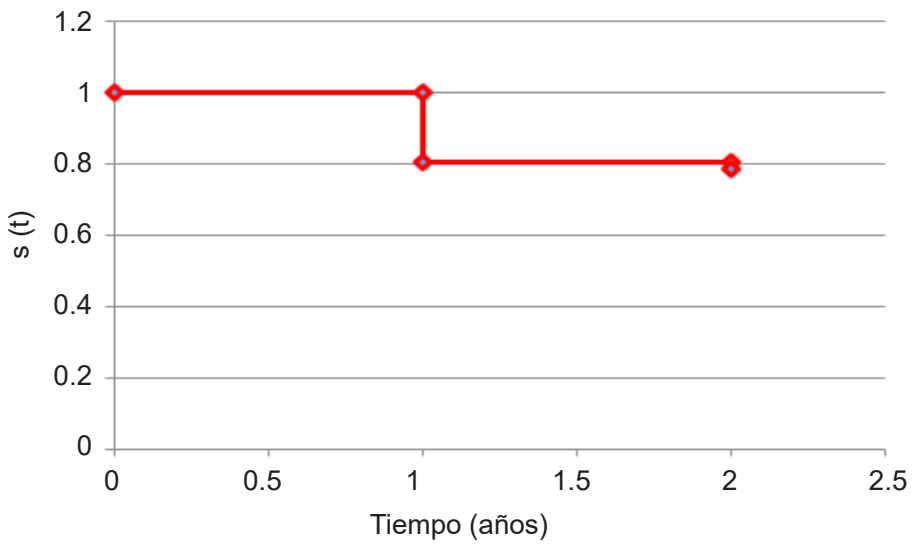

Fuente: Real Statitistics V7.6 Ene 2121, R V

Figura 2. Curva de supervivencia. 
de complicaciones posoperatorias como sepsis, peritonitis, fuga de la anastomosis, sangrado y mortalidad, así también como el nivel de hemoglobina y prealbúmina en el análisis exploratorio (tabla 8).

\section{Discusión}

Algunos estudios ${ }^{4-6}$ han indicado que la profundidad de invasión y la presencia o ausencia de metástasis ganglionares son los factores pronósticos más importantes en cáncer gástrico. Otros centran sus hipótesis en los marcadores tumorales que provienen principalmente del propio cáncer como factor pronóstico ${ }^{7}$.

Recientemente se le da valor pronóstico a los marcadores nutricionales como predictor de resultados en los pacientes con CG sometidos a gastrectomía total. Existen varias herramientas para evaluar el estado nutricional, entre las cuales las más simples y convenientes han sido clásicamente el Índice de Masa Corporal (IMC) y las proteínas séricas, como la albumina y la prealbúmina ${ }^{4-6}$.

Ouyang et $a l^{5}$ demostraron que los niveles de albúmina sérica preoperatoria, parámetro incluido en el INP, está asociada con el pronóstico en pacientes con CG. La albúmina ha sido comúnmente utilizada para determinar el estado nutricional de los pacientes y un estudio reveló que la albúmina sérica es un factor importante para predecir el pronóstico en pacientes con CG, sin embargo, el nivel de albumina sérica es una herramienta poco confiable ya que puede ser influenciado por reacciones inflamatorias sistémicas ${ }^{24}$. Así como en estos estudios, en el nuestro no tuvo valor predictivo como variable independiente.

Mantovani et al ${ }^{19}$ demostraron que la inflamación asociada a tumores se caracteriza por la proliferación de las células inflamatorias y la producción y liberación de factores inflamatorios en los tejidos tumorales. Las células inflamatorias y las células inmunes, como los neutrófilos, los

Tabla 8. Regresión logística para el índice nutricional pronóstico (INP), hemoglobina y prealbúmina.

\begin{tabular}{|c|c|c|c|c|}
\hline Complicación & & INP $>46,03$ & Hemoglobina $<10$ g/dl & Prealbúmina $<8 \mathrm{mg} / \mathrm{d}$ \\
\hline \multirow{3}{*}{ Sepsis } & Coeff b & $-0,18$ & $-1,56$ & $-0,13$ \\
\hline & Valor $p$ & 0,04 & 0,005 & 0,24 \\
\hline & $\mathrm{OR}\left(\mathrm{IC}_{95 \%}\right)$ & $\begin{array}{c}0,84 \\
(0,67-1,04) \\
\end{array}$ & $\begin{array}{c}4,77 \\
(1,61-14,1) \\
\end{array}$ & $\begin{array}{c}0,87 \\
(0,7-1,09) \\
\end{array}$ \\
\hline \multirow{3}{*}{ Peritonitis } & Coeff b & $-1,38$ & $-2,31$ & $-0,5$ \\
\hline & Valor $p$ & 0,001 & 0,04 & 0,1 \\
\hline & $\begin{array}{c}\text { OR } \\
\left(\mathrm{IC}_{95 \%}\right)\end{array}$ & $\begin{array}{c}0,9 \\
(0,5-0,97)\end{array}$ & $\begin{array}{c}10,05 \\
(8-20) \\
\end{array}$ & $\begin{array}{c}1,65 \\
(1,4-1,9)\end{array}$ \\
\hline \multirow{3}{*}{$\begin{array}{l}\text { Fuga de } \\
\text { la anastomosis }\end{array}$} & Coeff b & $-0,12$ & $-0,04$ & $-0,02$ \\
\hline & Valor $p$ & 0,01 & 0,08 & 0,02 \\
\hline & $\begin{array}{c}\text { OR } \\
\left(\mathrm{IC}_{95 \%}\right)\end{array}$ & $\begin{array}{c}0,88 \\
(0,77-1,01) \\
\end{array}$ & $\begin{array}{c}0,96 \\
(0,7-1,31) \\
\end{array}$ & $\begin{array}{c}1,98 \\
(1,2-2,11) \\
\end{array}$ \\
\hline \multirow{3}{*}{ Sangrado } & Coeff $b$ & $-2,15$ & $-0,71$ & $-4,8$ \\
\hline & Valor $p$ & 0,03 & 0,99 & 0,99 \\
\hline & $\begin{array}{c}\text { OR } \\
\left(\mathrm{IC}_{95 \%}\right)\end{array}$ & $\begin{array}{c}0,12 \\
(0,11-1,2)\end{array}$ & $\begin{array}{c}1,8 \\
(0,3-2,9)\end{array}$ & $\begin{array}{c}12,1 \\
(7-14)\end{array}$ \\
\hline \multirow{3}{*}{ Mortalidad } & Coeff b & $-4,61$ & $-0,45$ & $-1,77$ \\
\hline & Valor $p$ & 0,004 & 0,4 & 0,99 \\
\hline & $\begin{array}{c}\text { OR } \\
\left(I_{95 \%}\right)\end{array}$ & $\begin{array}{c}0,8 \\
(0,4-0,7)\end{array}$ & $\begin{array}{c}1,57 \\
(1,01-5)\end{array}$ & $\begin{array}{c}5,85 \\
(0,4-11,1)\end{array}$ \\
\hline
\end{tabular}


linfocitos y los monocitos, en la sangre periférica de los pacientes con inflamación asociada a un tumor, se consideran factores importantes que conducen al desarrollo, la invasión y la metástasis del tumor. Así como nuestro estudio, otros estudios han demostrado que los índices nutricionales y los parámetros inflamatorios juegan un papel importante en la determinación del pronóstico de los pacientes con cáncer ${ }^{31-33}$.

Debido a la mayor incidencia de CG, los países orientales han establecido programas de tamización, lo que conlleva a aumentar la detección de neoplasias en fases tempranas, con un mayor volumen de gastrectomías radicales y una menor morbimortalidad quirúrgica ${ }^{24}$. En contra parte, este estudio se ve limitado porque el CG potencialmente curable con manejo quirúrgico no es una entidad muy frecuente en nuestro medio.

En la literatura asiática, fundamentalmente en Japón, se han publicado varios trabajos ${ }^{24-26}$ que, como el nuestro, determinan el INP previo a la gastrectomía y estudian la posible relación entre un INP disminuido y la morbi-mortalidad postoperatoria. Aunque los resultados obtenidos no han sido concluyentes, de manera similar con los resultados de nuestro estudio, se plantea que la presencia de un INP bajo podría tener un valor predictivo sobre la frecuencia de complicaciones y supervivencia global. Sin embargo, existe la limitante porque es sabido que hay otros factores que también influyen en los resultados de los pacientes, como la quimioterapia y la parte psicológica, cuyo análisis no fue objetivo de este estudio ${ }^{24}$.

Identificando factores predictivos de morbilidad quirúrgica, se podría proporcionar un manejo perioperatorio individualizado, mejorando los resultados a corto y largo plazo en cuanto al periodo libre de enfermedad y la supervivencia general de los pacientes con cáncer ${ }^{24}$. En la práctica clínica, el INP puede, por lo tanto, servir para identificar a los pacientes con riesgo de mal pronóstico, predecir los sitios de recidiva de la enfermedad y vigilar metástasis hematógenas, e incluso para definir la indicación de la quimioterapia adyuvante en ciertos estadios en los cuales todavía hay discusión.
Nuestros resultados muestran que el INP se asocia con la mortalidad y complicaciones posoperatorias en pacientes sometidos a gastrectomía por cáncer gástrico, debido a que el INP puede reflejar el estado nutricional y la respuesta inflamatoria e inmunológica. Un INP preoperatorio alto en pacientes con CG sugiere que el soporte nutricional perioperatorio activo puede ser un nuevo método para mejorar los resultados de los pacientes.

En un estudio reciente ${ }^{24}$, la tasa de supervivencia postoperatoria de pacientes sometidos a gastrectomía radical con un INP menor de 46,03 fue significativamente menor que aquellos con INP mayor o igual a 46,03 $(23,7$ meses versus 34,3 meses; $p<0,001$ ), resultados que contrastan con los nuestros. A pesar de la mejoría de los cuidados perioperatorios y las técnicas quirúrgicas, la resección R0 del CG presenta una elevada frecuencia de complicaciones y mantiene una importante tasa de mortalidad. La morbilidad tras la gastrectomía radical es muy variable, con rangos que oscilan entre el 10,5 y el $46 \%$ y la mortalidad varía del 0,6 al 10,2 \% ${ }^{12,24,34}$.

La gran mayoría de los trabajos que analizan los posibles factores pronósticos en el CG se refieren a los casos en los que se ha practicado una gastrectomía aparentemente curativa (R0), por lo que su utilidad se ve limitada en el amplio porcentaje de pacientes en quienes no se practica una resección tumoral radical. En la actualidad, carecemos de datos suficientes en nuestro medio acerca de las variables con valor pronostico que puedan utilizarse en el momento del diagnóstico del tumor, que es cuando se debe tomar una decisión terapéutica ${ }^{12}$.

Las limitaciones de este estudio surgen de ser un análisis retrospectivo, con un tamaño de muestra pequeño; deben diseñarse estudios prospectivos, multicéntricos y a gran escala. Solo recopilamos datos sobre la supervivencia de los pacientes, pero no tuvimos acceso a datos completos y efectivos sobre la supervivencia libre de enfermedad, por lo tanto, no fue posible analizar estos importantes indicadores de pronóstico. 


\section{Conclusiones}

La alta frecuencia de muertes por CG en todo el mundo ha dirigido la mirada de los investigadores a encontrar estrategias para reducir la mortalidad. Son pocos los factores pronósticos que se han descrito en la literatura que afectan la supervivencia de los pacientes con CG. El principal de ellos es la cirugía, sin embargo, sigue teniendo una importante tasa de mortalidad y complicaciones asociadas que reducen la supervivencia y la calidad de vida de los pacientes.

Se hace necesario encontrar factores pronóstico antropométricos, clínicos y paraclínicos, que permitan establecer el riesgo en los pacientes con CG para definir el mejor tiempo quirúrgico y tipo de cirugía; entre estos, el estado nutricional es un factor pronóstico modificable con el tratamiento, que puede influir tanto en la morbilidad postoperatoria como en la supervivencia.

El índice nutricional pronóstico (INP) refleja el estado de inmunocompetencia y nutricional de los pacientes. Varios estudios, como el nuestro, han demostrado que los pacientes con bajo INP preoperatorio tienen pobre supervivencia, mayor mortalidad y complicaciones posoperatorias, al ser sometidos a gastrectomía por cáncer gástrico.

La identificación de factores predictivos para la morbilidad quirúrgica, posquirúrgicas y la presentación de complicaciones, es el nuevo reto de la investigación en cáncer gástrico, con el fin de encaminar mejor los planes perioperatorios individualizados a cada paciente.

\section{Cumplimiento de normas éticas}

Consentimiento informado. Se contó con la aprobación del comité de ética institucional. Los aspectos éticos se ajustaron a los principios éticos fundamentales, a las directrices de la Declaración de Helsinki, Asociación Médica Mundial, Informe Belmont, Pautas emanadas de la Asociación Médica Mundial y al artículo 11 de la Resolución 008430 del 4 de octubre de 1993 del Ministerio de Salud de Colombia.

Se solicitó consentimiento informado telefónico al paciente o su familiar cuando se realizó llamada telefónica para completar datos faltantes en la historia clínica del paciente.
Conflicto de intereses. Los autores declaran no tener ningún conflicto de interés.

Financiación. Este trabajo fue autofinanciado por los autores.

\section{Contribuciones de los autores}

- Concepción y diseño del estudio: Andrés Eduardo Marín-Castro, Douglas Omar Ortiz-Espinel, Carlos Alberto Sánchez-Toro, Carlos Manuel Zapata-Acevedo, María José Marín-Castro, Blas Darío Conde-Rodríguez.

- Adquisición de datos: Andrés Eduardo Marín-Castro, María José Marín-Castro, Blas Darío Conde-Rodríguez.

- Análisis e interpretación de datos: Andrés Eduardo Marín-Castro, Douglas Omar Ortiz-Espinel, Carlos Alberto Sánchez-Toro, Carlos Manuel Zapata-Acevedo, María José Marín-Castro, Blas Darío Conde-Rodríguez, Gerardo Ardila-Duarte.

- Redacción del manuscrito: Andrés Eduardo MarínCastro, Douglas Omar Ortiz-Espinel, Carlos Alberto Sánchez-Toro, Carlos Manuel Zapata-Acevedo, María José Marín-Castro, Blas Darío Conde-Rodríguez, Gerardo Ardila-Duarte.

- Revisión crítica: Douglas Omar Ortiz-Espinel, Carlos Alberto Sánchez-Toro, Carlos Manuel Zapata-Acevedo, Gerardo Ardila-Duarte.

\section{Referencias}

1. Oh SE, Choi MG, Seo JM, An JY, Lee JH, Sohn TS, et al. Prognostic significance of perioperative nutritional parameters in patients with gastric cancer. Clin Nutr. 2019;38:870-6. https://doi.org/10.1016/j.clnu.2018.02.015

2. Kanda M, Mizuno A, Tanaka C, Kobayashi D, Fujiwara M, Iwata $\mathrm{N}$, et al. Nutritional predictors for postoperative short-term and long-term outcomes of patients with gastric cancer. Medicine (Baltimore). 2016;95:e3781. https://doi.org/10.1097/MD.0000000000003781

3. Migita K, Matsumoto S, Wakatsuki K, Ito M, Kunishige T, Nakade $\mathrm{H}$, et al. A decrease in the prognostic nutritional index is associated with a worse long-term outcome in gastric cancer patients undergoing neoadjuvant chemotherapy. Surg Today. 2017;47:1018-26. https://doi.org/10.1007/s00595-017-1469-y

4. Yang Y, Gao P, Song Y, Sun J, Chen X, Zhao J, et al. The prognostic nutritional index is a predictive indicator of prognosis and postoperative complications in gastric cancer: A meta-analysis. Eur J Surg Oncol. 2016;42:1176-82.

https://doi.org/10.1016/j.ejso.2016.05.029

5. Ouyang X, Dang Y, Zhang F, Huang Q. Low serum albumin correlates with poor survival in gastric cancer patients. Clin Lab. 2018;64:239-45.

https://doi.org/10.7754/Clin.Lab.2017.170804 
6. Wang HX, Wang CC, Yang W, Gao LL, Yu SQ. Prognostic value of preoperative prognostic nutritional index in stage III gastric cancer after curative resection: a retrospective cohort study. Asia Pac J Clin Nutr. 2018;27:540-5.

https://doi.org/10.6133/apjcn.072017.03

7. Hirahara N, Tajima Y, Fujii Y, Kaji S, Yamamoto T, Hyakudomi R, et al. Prognostic nutritional index as a predictor of survival in resectable gastric cancer patients with normal preoperative serum carcinoembryonic antigen levels: a propensity score matching analysis. BMC Cáncer. 2018;18:285. https://doi.org/10.1186/s12885-018-4201-4

8. Gómez-Zuleta MA, Riveros-Vega JH, Ruiz O, Concha A, Ángel-Betancur DM, Torres-Amaya M, et al. Guía de práctica clínica para la prevención, diagnóstico y tratamiento del cáncer gástrico temprano - 2015. Rev Col Gastroenterol. 2015;30(suppl.1):34-42.

9. Siegel RL, Miller KD, Jemal A. Cancer statistics, 2018. CA Cancer J Clin. 2018;68:7-30. https://doi.org/10.3322/caac.21442

10. Markar SR, Karthikesalingam A, Jackson D, Hanna GB. Long-term survival after gastrectomy for cancer in randomized, controlled oncological trials: comparison between West and East. Ann Surg Oncol. 2013;20:2328-38. https://doi.org/10.1245/s10434-012-2862-9

11. Karimi P, Islami F, Anandasabapathy S, Freedman ND, Kamangar F. Gastric cancer: descriptive epidemiology, risk factors, screening, and prevention. Cancer Epidemiol Biomarkers Prev. 2014 May;23(5):700-13. https://doi.org/10.1158/1055-9965.EPI-13-1057

12. Borda A, Borda F, Vila J, Fernández-Urién I, Zozaya JM, Guerra A. Valor predictivo pre-tratamiento del Índice Pronóstico Nutricional sobre la supervivencia del carcinoma gástrico. An Sist Sanit Navar. 2016;39:227-35. https://doi.org/10.23938/ASSN.0271

13. Viúdez-Berral A, Miranda-Murua C, Arias de la Vega F, Hernández-García I, Artajona-Rosino A, Díaz de Liaño A, Vera-García R. Situación actual en el tratamiento del cáncer gástrico. Rev Esp Enf Dig. 2012;104:134-41. https://doi.org/10.4321/S1130-01082012000300006

14. Gullo I, Carneiro F, Oliveira C, Almeida GM. Heterogeneity in gastric cancer: From pure morphology to molecular classifications. Pathobiology. 2018;85:50-63. https://doi.org/10.1159/000473881

15. Fukuda Y, Yamamoto K, Hirao M, Nishikawa K, Maeda $\mathrm{S}$, Haraguchi N, et al. Prevalence of malnutrition among gastric cancer patients undergoing gastrectomy and optimal preoperative nutritional support for preventing surgical site infections. Ann Surg Oncol. 2015;22 Suppl 3:S778-85. https://doi.org/10.1245/s10434-015-4820-9

16. Ryu SW, Kim IH. Comparison of different nutritional assessments in detecting malnutrition among gastric cancer patients. World J Gastroenterol. 2010;16:3310-7. https://doi.org/10.3748/wjg.v16.i26.3310
17. Fujiya K, Kawamura T, Omae K, Makuuchi R, Irino T, Tokunaga $\mathrm{M}$, et al. Impact of malnutrition after gastrectomy for gastric cancer on long-term survival. Ann Surg Oncol. 2018;25:974-83. https://doi.org/10.1245/s10434-018-6342-8

18. Balkwill F, Mantovani A. Inflammation and cancer: Back to Virchow? Lancet. 2001;357:539-45. https://doi.org/10.1016/S0140-6736(00)04046-0

19. Mantovani A, Allavena P, Sica A, Balkwill F. Cancer-related inflammation. Nature. 2008;454:436-44. https://doi.org/10.1038/nature07205

20. Labelle M, Begum S, Hynes RO. Direct signaling between platelets and cancer cells induces an epithelial-mesenchymal-like transition and promotes metastasis. Cancer Cell. 2011;20:576-90. https://doi.org/10.1016/j.ccr.2011.09.009

21. Zhang X, Shi H, Yuan X, Jiang P, Qian H, Xu W. Tumor-derived exosomes induce N2 polarization of neutrophils to promote gastric cancer cell migration. Mol Cancer. 2018;17:146. https://doi.org/10.1186/s12943-018-0898-6

22. Pylaeva E, Harati MD, Spyra I, Bordbari S, Strachan S, Thakur BK, et al. NAMPT signaling is critical for the proangiogenic activity of tumor-associated neutrophils. Int J Cancer. 2019;144:136-49. https://doi.org/10.1002/ijc.31808

23. Huang C, Li Z, Li N, Li Y, Chang A, Zhao T, et al. Interleukin 35 expression correlates with microvessel density in pancreatic ductal adenocarcinoma, recruits monocytes, and promotes growth and angiogenesis of xenograft tumors in mice. Gastroenterology. 2018;154:675-88. https://doi.org/10.1053/j.gastro.2017.09.039

24. Saito H, Kono Y, Murakami Y, Kuroda H, Matsunaga T, Fukumoto Y, Osaki T. Influence of prognostic nutritional index and tumor markers on survival in gastric cancer surgery patients. Langenbecks Arch Surg. 2017;402:501-7. https://doi.org/10.1007/s00423-017-1572-y

25. Rho SY, Hwang HK, Chong JU, Yoon DS, Lee WJ, Kang CM. Association of preoperative total lymphocyte count with prognosis in resected left-sided pancreatic cancer. ANZ J Surg. 2019;89:503-8. https://doi.org/10.1111/ans.15030

26. Noh GT, Han J, Cho MS, Hur H, Min BS, Lee KY, Kim NK. Impact of the prognostic nutritional index on the recovery and long-term oncologic outcome of patients with colorectal cancer. J Cancer Res Clin Oncol. 2017;143:1235-42. https://doi.org/10.1007/s00432-017-2366-x

27. Wang Z, Wang J, Wang P. The prognostic value of prognostic nutritional index in hepatocellular carcinoma patients: A meta-analysis of observational studies. PLoS One. 2018;13:e0202987. https://doi.org/10.1371/journal.pone.0202987

28. Li S, Tian G, Chen Z, Zhuang Y, Li G. Prognostic role of the prognostic nutritional index in pancreatic cancer: A meta-analysis. Nutr Cancer. 2019;71:207-13. https://doi.org/10.1080/01635581.2018.1559930 
29. Onodera T, Goseki N, Kosaki G. Prognostic nutritional index in gastrointestinal surgery of malnourished cancer patients. Nihon Geka Gakkai Zasshi. 1984;85:1001-5.

30. Wang L, Miao Y, Chen T, Sun D, Ge S, Zuo L, Liu M. Value of the preoperative prognostic nutritional index for the evaluation of patient prognosis after radical gastrectomy. Mol Clin Oncol. 2020;12:196-201.

https://doi.org/10.3892/mco.2020.1980

31. Inoue D, Sekiguchi S, Yamagata W, Maeda G, Yamada D, Fujiwara S, et al. Elevation of neutrophil-to-lymphocyte ratio before first-line chemotherapy predicts a poor prognosis for second-line chemotherapy in gastric cancer. Oncology. 2019;96:140-6. https://doi.org/10.1159/000493427

32. Szor DJ, Roncon-Dias A, Pereira MA, Ramos MFKP, Zilberstein B, Cecconello I, Ribeiro U. Neutrophil-lympho- cyte ratio is associated with prognosis in patients who underwent potentially curative resection for gastric cancer. J Surg Oncol. 2018;117:851-7. https://doi.org/10.1002/jso.25036

33. Pan YC, Jia ZF, Cao DH, Wu YH, Jiang J, Wen SM, et al. Preoperative lymphocyte-to-monocyte ratio (LMR) could independently predict overall survival of resectable gastric cancer patients. Medicine (Baltimore). 2018;97:e13896.

https://doi.org/10.1097/MD.0000000000013896

34. Borda F, Miranda C, Borda A, Echeverría E, Guerra A, Iñigo JJ, Zozaya JM. Relación entre el índice de Onodera pre-operatorio y las complicaciones post-cirugía R0 en el cáncer de estómago. An Sist Sanit Navar. 2017;40:67-75. https://doi.org/10.23938/ASSN.0007 